\title{
A SIMPLER PROOF OF MIRZAKHANI'S SIMPLE CURVE ASYMPTOTICS
}

\author{
IGOR RIVIN
}

\begin{abstract}
Aвstract. Maryam Mirzakhani (in her doctoral dissertation) has proved the author's conjecture that the number of simple closed curves of length bounded by $L$ on a hyperbolic surface $S$ is asymptotic to a constant times $L^{d}$, where $d$ is the dimension of the Teichmüller space of $S$. In this note we clarify and simplify Mirzakhani's argument.
\end{abstract}

\section{INTRODUCTION}

In [7] M. Mirzakhani shows that the number of closed geodesics of length bounded above by $L$ in the mapping class group orbit of a fixed curve $\gamma$ on a hyperbolic surface $X$ grows like a (positive) constant times $L^{\operatorname{dim} T(X)}$, where $T(X)$ is the Teichmüller space of $X$. This result, together with the obvious (but easy to miss) observation that simple curves fall into a finite number of orbits, implies my conjecture that the number $N_{L}(X)$ of simple closed geodesics of length bounded above by $L$ has the same order of growth. Such a result was obtain by G. McShane and the author in [6, [5] in the special case where $X$ is homeomorphic to a punctured torus. In the general case, I had previously obtained an order of growth result in [9]. The approach of [9] was largely combinatorial, and Mirzakhani has brought a number of different ideas to bear on the question. These ideas are:

(1) One should study the orbit of a fixed curve.

(2) One can get a lot of H. Masur's result [4]: the action of the mapping class group on the space of measured laminations of a surface is ergodic.

Date: February 2, 2008.

1991 Mathematics Subject Classification. 57M50, 32G15.

Key words and phrases. hyperbolic structures, mapping class group, ergodicity, Mirzakhani, simple curves.

The author thanks Greg McShane for useful conversations, and Benson Farb on helpful comments on a previous draft of this paper. 
(3) The growth rate is the product of two factors: one depends on the hyperbolic metric on $X$, while the other does not. One can then "separate variables" by integrating over moduli space.

(4) The last integration uses Mirzakhani's result that the WeilPetersson volumes of moduli spaces of surfaces with boundary are polynomials in the length of the boundary.

In this note, we combine these ideas with the results of [9], the coarea formula, and some simple observations to simplify Mirzakhani's argument.

\section{Multicurves}

The first part of the argument concerns multicurves on a hyperbolic surface $S$. A multicurve is a collection of pairwise disjoint simple closed curves on $S$, together with the assignment of an integer weight to each curve in the collection (the weight is usually thought of as the number of times the curve winds around itself).

There is a way to parametrize the set of all multicurves (discovered by Max Dehn): we decompose $S$ (in some fixed way) into pairs of pants, then record, for each pants boundary curve, how many times the multicurve intersects it and how many times it winds around it. Winding can be clockwise or counterclockwise, while intersection numbers are geometric intersection numbers, and so are positive (and also even). It is an easy result of Dehn's that there is a bijective corespondence between the collections of such integer coordinates and multicurves; the reader can consult Dehn's original paper [1] or the more recent [8] for the proofs of the assertions above.

Dehn's coordinates thus represent multicurves as integer lattice points in an improper cone in $\mathbb{R}^{\operatorname{dim} \mathcal{M L}(S)}{ }^{1}$ This cone can be thought of as the measured lamination space $\mathcal{M L}$ - that identification is the content of Theorem 3.1.1 in [8]. Explicitely

$$
\mathcal{M} \mathcal{L}(S)=\mathbb{R}^{(\operatorname{dim} \mathcal{M L}) / 2} \times \mathbb{R}_{+}^{(\operatorname{dim} \mathcal{M L}) / 2},
$$

where the first half of the coordinates correspond to twists, and the second to the intersection numbers; multicurves correspond to points in $\mathcal{M L}(S)$ where the first half of the coordinates are integral, and the second half are even.

\footnotetext{
${ }^{1}$ For a closed surface of genus $g$, the dimension of the moduli space (and of the measured lamination space) is $6 g-6$, for a surface with $c$ punctures and $p$ perforations, the dimension is $6 g-6+2 c+2 p$. Since none of the arguments below depend on the signature of the surface, we will always denote the dimension by $\operatorname{dim} \mathcal{M L}(S)$ or $\operatorname{dim} \mathbb{M}(S)$, (the two are equal) as appropriate.
} 
On this cone we have a measure, induced from Lebesgue measure in the ambient $\mathbb{R}^{n}$. As is well known to physicists far and wide, there is another way to define the Lebesgue measure of an open set $\Omega$ in $\mathbb{R}^{n}$ :

$$
\lambda(\Omega)=\lim _{t \rightarrow \infty} \frac{\left|t \Omega \cap \mathbb{Z}^{n}\right|}{t^{n}} .
$$

This is just Riemann integration in $\mathbb{R}^{n}$. For future reference, let us also define

$$
\lambda_{t}(\Omega)=\frac{\left|t \Omega \cap \mathbb{Z}^{n}\right|}{t^{n}}
$$

so that

$$
\lambda(\Omega)=\lim _{t \rightarrow \infty} \lambda_{t}(\Omega) .
$$

It follows from these definitions that the Lebesgue measure is invariant under the Mapping Class Group of $S$, (henceforth denoted by $\operatorname{Mod}(S)$, since $\operatorname{Mod}(S)$ acts bijectively on integer lattice points and respects scaling.

\section{LENGTH FUNCTIONS}

Dehn's coordinates are essentially topological, but a hyperbolic structure on a surface $S$ defines a length function on multicurves, where the length of a multicurve is simply the weighted sum of the lengths of the geodesic representatives of the connected components of the multicurve in question. Indeed, one way to think of the length is as the inf of the lengths of topological (multi)curves isotopic to the given multicurve. It is then clear that the length function is linear on rays (this follows from the uniqueness part of Dehn's theorem alluded to above) and otherwise is convex (since $L(a+b) \leq L(a)+L(b))$. The length function can thus be extended by linearity to all points with rational coordinates, and by continuity (which follows from the triangle inequality above) to all real points in $\mathcal{M L}$ (This construction is identical to and slightly more general than the construction in [6, 5], as is the immediate sequel). From the convexity of the length function $L$ it follows that the set

$$
B_{L}(1)=\{x \mid L(x)<1\}
$$

is a convex set in $\mathcal{M} \mathcal{L}$. Further, by linearity and the definition of the measure $\lambda$ in Section 1, it follows that

Theorem 1. The number of multicurves of length bounded above by $L$ is asymptotic to

$$
\lambda\left(B_{L}(1)\right) L^{\operatorname{dim} \mathcal{M L}}
$$


Theorem 1 would be useless, unless we knew that

$$
0<\lambda\left(B_{L}(1)\right)<\infty .
$$

Luckily, that is well-known, and follows from the fact that the $L^{1}$ norm of the Dehn coordinate vector is quasi-the-same as the hyperbolic length of the corresponding curve (the distortion depends on the hyperbolic structure, and diverges as the hyperbolic structure goes to the boundary of Teichmüller space). Essentially that result is explained in [9], though there is no doubt tha the result was known to experts for at least twenty years beforehand.

An additional observation is that $\lambda\left(B_{L}(1)\right)$ varies analytically over moduli space. This follows, eg, from the methods of [3].

\section{Orbit DENSity}

Consider a set $X \subseteq \mathcal{M} \mathcal{L}$ and a multicurve $x \in \mathcal{M} \mathcal{L}$. Let $O(x)$ denote the orbit of $x$ under the mapping class group, and define

$$
\mu_{t}(X)=t^{-\operatorname{dim} \mathcal{M L}}|O(x) \cap t X| .
$$

Each $\mu_{t}$ defines a $\operatorname{Mod}(S)$-invariant measure, and it is clear that the family $\left\{\mu_{t}\right\}$ is bounded, thus weakly compact. Since $\mu_{t}(X) \leq \lambda_{t}(X)$ for all $t$, it follows that for any subsequence $\sigma$, the subsequence limit $\mu_{\sigma}$ is absolutely continuous with respect to $\lambda$. Since the action of $\operatorname{Mod}(S)$ on $\mathcal{M L}$ is ergodic, it follows that such a subsequence limit $\mu_{\sigma}$ is a constant multiple of $\lambda$. That is, for any finite measurable $X$,

$$
\mu_{\sigma}(X)=c_{\sigma}(x) \lambda(X) \text {. }
$$

In particular, we can take $X=B_{L}(1)$ for a length function $L$ coming from a hyperbolic structure on $S$. The results of [9] give the following

Theorem 2. There exists $a$ curve $x$, and a constant $c$ such that $c_{\sigma}(x)>c$ for any subsequence $\sigma$.

Proof. I show in [9] that the number of simple curves of length bounded by $L$ has order of growth $L^{\operatorname{dim} \mathcal{M L}}$. Since there is only a finite number of $\operatorname{Mod}(S)$ orbits of simple closed curves, at least one of the orbits grows at that speed, and this gives the desired $x$.

\section{Curves And moduli}

Take a surface $S$ and a curve $e^{2} \gamma$. Take the cover $\mathbb{M}^{\gamma}$ of the moduli space $\mathbb{M}(S)$ corresponding to the subgroup $\operatorname{Stab}(\gamma) \subset \operatorname{Mod}(S)$. If we

\footnotetext{
${ }^{2}$ There is no need for $\gamma$ to be a curve - all of the arguments work mutatis mutandis for a disjoint collection of essential curves. The argument does not work directly for general multicurves, though the result for such is easily deduced.
} 
cut $S$ along $\gamma$, we obtain a (possibly disconnected) surface $S^{\prime}$ with two boundary components of the same length, and it is clear that this induces a map $\pi_{\gamma}: \mathbb{M}^{\gamma} \rightarrow \mathbb{M}_{\gamma} S^{\prime}$, where the last subscript indicates that two of the boundary components correspond to the same curve. The map is a fibration, where the fiber corresponds to twisting along $\gamma$. If we take a pair of pants decomposition of $S$ which includes $\gamma$ as one of the pants curves, $\pi$ acts very simply on the Weil-Petersson symplectic form: the term $d \ell(\gamma) \wedge d \tau(\gamma)$ is simply dropped. The fiber is a circle of length $L(\gamma)$. This shows (by the coarea formula (Corollary 6) that the volume of that piece of $\mathbb{M}^{\gamma}$ where the length of the appropriate translate of $\gamma$ equals $l$ is simply $l$ times the volume of the moduli space of $S^{\prime}$ where the two curves corresponding to $\gamma$ have length $l$. This is so because Wolpert's formula (see [10]) implies that the Jacobian of $\pi_{\gamma}$ equals 1 .

We remind the reader that Wolpert's formula for the Weil-Petersson Kähler form reads:

$$
W P=\sum_{\gamma} d \ell(\gamma) \wedge d \tau(\gamma)
$$

where the sum is taken over all curves in a pants decomposition of $S, \ell(\gamma)$ is the length of $\gamma$ and $\tau(\gamma)$ is the twist along $\gamma$.

Let us now denote the number of curves in the $\operatorname{Mod}(S)$ orbit of $\gamma$ of length not exceeding $L$ (corresponding to a hyperbolic structure $\mathcal{H}$ ) by $n_{\mathcal{H}}(L)$, and let us ask what the average value of $n_{\mathcal{H}}(L)$ is over the moduli space of $S$. It turns out to be easier to not normalize by the volume of $\mathbb{M}(S)$. In that case, we have the following obvious relationship:

$$
\int_{\mathbb{M}(S)} n_{\mathcal{H}(L)}=\operatorname{Vol}\left\{(x, \rho) \in \mathbb{M}^{\gamma} \mid \quad L(\rho)<L\right\},
$$

where the notation $(x, \rho)$ means that we are in the sheet corresponding to the image $\rho$ of $\gamma$. The volume in the right hand side of the Eq. (1) is easy to evaluate with the help of the discussion at the beginning of the section, the coarea formula, and Mirzakhani's results on the Weil-Petersson volumes of moduli spaces of bordered surfaces: the answer is a polynomial of $\operatorname{degree} \operatorname{dim} \mathbb{M}(S)=\operatorname{dim} \mathcal{M} \mathcal{L}$. This gives us the following observation:

Lemma 3. There exists a constant $C$, such that

$$
L^{-\operatorname{dim} \mathbb{M}(S)} \int_{\mathbb{M}(S)} n_{\mathcal{H}(L)}<C .
$$


Now we can show that the constant $c_{\sigma}(x)$ in Section 3 does not depend on the subsequence $\sigma$. Indeed, with the limit being taken over an arbitrary subsequence $\sigma$ :

$$
\lim \int_{\mathbb{M}(s)} n_{\mathcal{H}(L)} L^{-\operatorname{dim}(\mathbb{M}(S))}=\int_{\mathbb{M}(S)} \lim n_{\mathcal{H}(L)} L^{-\operatorname{dim}(\mathbb{M}(S)),}
$$

by the Dominated Convergence Theorem and Lemma 3. The left hand side does not depend on the subsequence by the discussion following Eq. (11). The right hand side equals

$$
\int_{\mathbb{M}(S)} c_{\sigma}(\gamma) \lambda\left(B_{L}(1)\right)
$$

and so also does not depend on the choice of the subsequence $\sigma$.

Note that if we pick $\gamma$ to be the curve whose existence is shown in Theorem 2, it follows that:

Theorem 4. The integral of $\lambda\left(B_{L}(1)\right)$ over $\mathbb{M}(S)$ is finite.

It then follows immediately that $c_{\sigma}(\gamma)$ is itself independendent of $\sigma$.

\section{THE COAREA FORMULA}

In this setting we need a very simple version of the coarea formula of Federer, but we shall state a more general version. Our source for this is Ralph Howard's exposition [2]. First, $J f$ is the Jacobian of $f$, defined (under the assumption that $m \geq n$ ) as:

$$
J f(x)= \begin{cases}0, & \text { if } x \text { is a critical point of } f, \\ \sqrt{\operatorname{det}\left(f_{*}(x) f_{*}^{t}(x)\right)}, & \text { otherwise. }\end{cases}
$$

Theorem 5 (The Coarea Formula). Let $f: M^{m} \rightarrow N^{n}$ be a smooth map between Riemannian manifolds, with $m \geq n$. Then, for almost every $y \in N^{n}$, the fiber $f^{-1}(y)$ is either empty or a submanifold of $M^{m}$ of dimension $m-n$. For each regular value $y$ of $f$, let $d A$ be the $m-n$ dimensional surface area measure on $f^{-1}(y)$. Then, for any measurable function $h$ on $M^{m}$,

$$
\int_{N^{n}} \int_{f^{-1}(y)} h d A d Y=\int_{M^{m}} h(x) J f(x) d x,
$$

where $d y$ is the Riemannian volume measure on $N^{n}$, and $d x$ is the Riemannian volume measure on $M^{m}$.

Corollary 6. Let $\mathcal{H}^{m-n}\left(f^{-1}(y)\right)$ denote the $m-n$ dimensional area of $f^{-1}(y)$. Then

$$
\int_{N^{n}} \mathcal{H}^{m-n}\left(f^{-1}(y)\right) d y=\int_{M^{m}} J f(x) d x .
$$


Proof. Set $h \equiv 1$ in Theorem 5

\section{REFERENCES}

[1] Max Dehn. Lecture notes from breslau. Technical report, 1922. Archive of the University of Texas at Austin.

[2] Ralph Howard. The kinematic formula in Riemannian homogeneous spaces. Mem. Amer. Math. Soc., 106(509):vi+69, 1993.

[3] Steven P. Kerckhoff. Earthquakes are analytic. Comment. Math. Helv., 60(1):1730, 1985.

[4] Howard Masur. Ergodic actions of the mapping class group. Proc. Amer. Math. Soc., 94(3):455-459, 1985.

[5] Greg McShane and Igor Rivin. Geometry of geodesics and a norm on homology. International Mathematics Research Notices, (2):61-69, February 1995.

[6] Greg McShane and Igor Rivin. Simple curves on hyperbolic tori. Comptes Rendus Acad. Sci. Paris, Sér. I. Math, 320(12):1523-1528, June 1995.

[7] Maryam Mirzakhani. Growth of the number of simple closed geodesics on hyperbolic surfaces. submitted, 2004.

[8] R. C. Penner and J. L. Harer. Combinatorics of train tracks, volume 125 of Annals of Mathematics Studies. Princeton University Press, Princeton, NJ, 1992.

[9] Igor Rivin. Simple curves on surfaces. Geometriae Dedicata, 87(1/3):345-360, August 2001.

[10] Scott Wolpert. On the symplectic geometry of deformations of a hyperbolic surface. Ann. of Math. (2), 117(2):207-234, 1983.

Department of Mathematics, Temple University, Philadelphia

E-mail address: rivin@math.temple.edu 